\title{
WE BELONG TOGETHER? A PLEA FOR MODESTY IN MODAL PLURAL LOGIC
}

\author{
SIMON HEWITT
}

'We belong together', a doey eyed lover says to his beloved. She, being both better educated in a certain sort of metaphysics than her suitor and more romantic, responds 'Yes. Not only in the actual world, but in every possible world. There is no possible world in which we are not together'. Whether this is genuinely touching or simply emetic is a fine judgement. Perhaps more remarkably this expression of lovestruck hyperbole bears a striking similarity to a principle enshrined as current orthodoxy in discussions of the modal logic of plurals. Let $x x$ be some things; then, goes one half of the claim, if $x$ is one of $x x$ then necessarily: if $x x$ exist, then $x$ is one of $x x$. Similarly, if $x$ is not one of $x x$ then necessarily: if $x x$ exist, then $x$ is not one of $x x$. The path from love to logic is a short one indeed.

Why should this be of any more than esoteric interest? The principle, which sometimes gets called plural rigidity ${ }^{1}$ is put to work to important and controversial metaphysical ends. Crucially, Williamson deploys it in arguing both for necessitism (the doctrine that ontology is modally invariant) and for a property-based interpretation of second-order quantification (since he thinks the modal behaviour of plurals rules out a Boolos style plural interpretation) ([25]) ([24]) ([4]). Linnebo develops a foundational programme for set-theory in a modal plural logic strengthened by the addition of statements equivalent to the principle, and whilst he himself proposes a non-standard interpretation of the modalities, its acceptablity with respect to metaphysical modality would provide a fall-back position for someone sympathetic to the approach ([12]).

A number of current philosophical projects, then, turn on this particular claim about the modal behaviour of pluralities. ${ }^{2}$ Yet it is often adopted swiftly without the sustained argument which might

Thanks to audiences at the Second Workshop of the European Non-Categorical Thinking Network, Turin, and in Leeds, and to John Divers, Keith Hossack and - especially - Øystein Linnebo for discussions of material relevant to this paper. The research leading to this paper was part of the ERC Nature of Representation project at the University of Leeds, grant number 312938.

${ }^{1}$ I will object to this usage below.

${ }^{2}$ Here, and throughout, 'plurality' is a convenient singularising locution, as is standard in the literature: a plurality is some things. 
reasonably be required to sustain a claim with such wide-ranging consequences. ${ }^{3}$ Recently, Linnebo has made in the direction of making good this deficiency ([13]). The present paper argues that Linnebo fails to supply sufficient reasons to adopt the claim at issue, and that in the absence of alternative arguments a question mark must hang over standard appeals to strong modal logics of plurals by philosophers. Of necessity, given the current state of the literature and the importance of Linnebo's work, much of what follows consists of a close criticism of Linnebo, but I go on to suggest that progress in the debate requires us to get clear about the relationship between language and non-linguistic reality and to pay close attention to linguistic use. These thoughts look likely to generalise across the philosophy of modal logic and to call into question much of the present fashion for modalising.

I will assume a working knowledge of customary notation and formation rules for plural logic. Good introductions are available in the form of [11] and [18]. A brief overview is provided here in an appendix. Whilst it is standard to offer informal interpretations of modal languages in terms of possible-worlds, as the appendix here does for the sake of brevity, the metaphysical associations of this are unhelpful in the present context and present the danger of building in a bias towards semantic realism which sits uncomfortably with suggestions I want to make. I will therefore deploy the less-worn language of truth with respect to possibilities or else simply interpret modal operators homophonically. After all, if we do not have a working understanding of expressions such as 'necessarily' and 'possibly' what do we take modal logics to be interpreting?

The paper proceeds as follows. $\S 1$ lays out the issue to be discussed, and undermines initial motivation for it. $\S \S 2-4$ work through Linnebo's formal arguments, diagnosing a common justificatory circularity. $\$ 5$ situates the sceptical position thus arrived at methodologically. I urge caution in the invocation of such a strong modal claim for philosophical argument in the absence of more persuasive arguments for its truth. I suggest that more attention needs to be paid to the use of language in

\footnotetext{
${ }^{3}$ An earlier attempt to remedy this situation is [23].

${ }^{4}$ I take 'modal logic' here to have the sense customary in philosophy, where interpretation is restricted to alethic, and in particular often metaphysical, modalities. Of course, considered as mathematical formalisms, modal logics are interesting in themselves and have a wide variety of applications. For a survey and introduction see [1]. Regarding interpretation, I find the suggestion that Kripke semantics - undoubtedly useful for simplifying proof technique, through construction of countermodels and the like - shed light on hitherto unanswered questions about the meaning of modal operators quite incredible. Nonetheless, my impression is that it is part of philosophical folklore. It is made explicit at [17, 121].
} 
making assertitions and performing deductions as a key, and perhaps the only, constraint on deciding the acceptability of candidate logical principles. This position looks likely to have implications beyond the present case, and to call into question standardly accepted modal axioms.

What Linnebo terms plural rigidity, we will call plural necessitism. The expression is still not perfect (it might suggest a deeper association with Williamson's metaphysics than can be supported), but at least avoids the danger of use-mention confusion that attaches to using the word 'rigidity' of a principle stated in the material, rather than formal, mode. ${ }^{5}$ Plural necessitism is equivalent to the conjunction of:

(NecInc)

$$
x<y y \rightarrow \square(E x \wedge E y y \rightarrow x<y y)
$$

(NecNInc)

$$
x \nless y y \rightarrow \square(E x \wedge E y y \rightarrow x \nprec y y)
$$

Here ' $E$ ' is to be read as an existence predicate, ${ }^{6}$ allowing us to work within a variable domain framework. It is routine to modify (NecInc) and (NecNInc) for a fixed domain framework simply by removing the second conditional along with its antecedent conjunction in each formula. In what follows, versions of the principles with and without existence predicates will be denoted by '(NecInc)' and '(NecNInc)', with context serving to disambiguate.

As we have seen, plural necessitism plays a non-negligible part in motivating or supporting metaphysically contentious positions. What reason, then is there to believe that it is true? It is provably independent of any normal modalisation of PFO+ ([10]). Motivation will therefore have to take the form of formal argument from independently compelling premises or of informal arguments.

As advertised above, our engagement will be mainly with the formal arguments Linnebo offers in support of plural necessitism (hereafter pn). Before that, some comment should be made on a

\footnotetext{
${ }^{5}$ Linnebo himself is keenly aware of this danger $([13,7])$.

${ }^{6}$ Strictly speaking, we may need to be more careful. We have not formally specified a lexicon, and in particular have not settled the question whether predicates may be untyped, in the sense that they may have argument places for which either singular or plural terms are eligible. From the perspective of tractable formation rules, proof theory and model theory, it is likely to be easier to type predicates. If the lexicon is typed, then ' $E$ ' is doing illegitimate double work in (NecInc) and (NecNInc) as formulated. We can get round this straightforwardly, by using superscripts to signify the type of existence predicates in the formal specification of the lexicon, and then omit superscripts in practice as a harmless convention.
} 
case for the plausibility of $p n$ which Linnebo develops by analogy with an argument for the modal invariance of set membership, based on extensionality and the necessity of identity. ${ }^{7}$ This proceeds from the principle:

(Indisc)

$$
x x \equiv y y \rightarrow(\phi(x x) \leftrightarrow \phi(y y))
$$

The subformula ' $x x \equiv y y$ ' abbreviates ' $\forall u(u<x x \leftrightarrow u<y y)$ '. This relieves us from the need to read ' $\equiv$ ' as denoting identity on pluralities. This is dialectically useful, since the extent of the similarity of behaviour (in modal contexts) between the referrents of singular and of plural terms is what is at issue. We do not therefore want to commit ourselves up front to a plural identity relation, since doing so might seem to presuppose that pluralities are object-like. From (Indisc) is derivable: ${ }^{8}$

$$
x x \equiv y y \rightarrow \square(x x \equiv y y)
$$

'(Cov)' here stands for covariance, the thought being that actually coextensive pluralities covary modally. Now (Cov) does not entail pn, since it is compatible with pluralities 'drifting' modally. Suppose that, with respect to some possibility $w_{1}, x x$ just are $y y$. In order for (Cov) to be satisfied, we require that at any possibility accessible relative to $w_{1}, x x$ just are $y y$. But now consider $w_{2}$, accessible relative to $w_{1}$, such that $x x$ and $y y$ both consist of the members of those pluralities with respect to $w_{1}$ with the addition of $a$, which does not exist with respect to $w_{1}$. We have here no counter-example to (Cov), but pn fails. ${ }^{9}$

Linnebo takes this drift to be indicative of soft extensionalism about pluralities. This is, the doctrine that extensionality holds within possibilities but not across them. He argues that this is an unstable position, for the same reason that he holds the parallel position regarding sets to be unsuccessful. With respect to the latter, he draws a comparison between sets and groups. We might think

\footnotetext{
${ }^{7}$ The case for the necessity of identity rehearsed by Linnebo is that developed by Barcan Marcus in ([15]).

${ }^{8}$ Proof sketch: (1) $x x \equiv y y$ (Ass for CP), (2) $\phi(x x) \equiv \phi(y y)((1$, Indisc) $),(3)(x x \equiv x x) \leftrightarrow \square(x x \equiv x x)($ Thm $),(4)(x x \equiv y y) \leftrightarrow$ $\square(y y \equiv x x)(3,2),(5) \square(y y \equiv x x)(1,4, \mathrm{MP}),(6) x x \equiv y y \rightarrow \square(x x \equiv y y)(1-5, \mathrm{CP})$

${ }^{9}$ Compare the counter-model to (NecInc) provided (within a fixed domain semantics) in ([10]).
} 
of groups as akin to social entities, such as the Supreme Court justices or the Celtic first eleven: ${ }^{10}$

The only reason to accept the principle of extensionality [for sets]... is that a set, unlike a group, is fully specified by its elements. Thus, when tracking a set across possible worlds, there is nothing other than the elements to go on. This ensures that the tracking is rigid. By contrast, when tracking a group, there is more than the members to go on... These considerations give rise to a dilemma that applies not only to sets but to any other notion of collection: either we have to give up the principle of extensionality, or else we have to accept the rigidity principle. There is no stable middle ground. Kripke famously taught us that there can be no 'soft identity theory' in the philosophy of mind, according to which mental states are identical with physical ones, but only contingently so, only the 'hard identity theory' committed to necessary identity. Our present conclusion is analogous. There can be no 'soft extensionalism' concerning sets of other kinds of collection, only 'hard extensionalism' that incorporates the rigidity claims and the idea of transworld extensionality that they embody. $([13,6])$

This is surely correct as regards sets: as Boolos once put the point, if anything deserves to be called analytic of our concept of set it is the axiom of extensionality $([2]$,$) , and the move from this$ recognition to the claim that possession of all and only its actual elements is an essential property of any given set is a natural one. The present issue, however, is whether this form of reasoning transfers across to the plural case. And here a number of questions arise. Most fundamentally, it is not entirely obvious that pluralities need be regarded as extensional in any sense: the temptation to think this as immediate as it is in the set-theoretic case arises from the truism 'a plurality just is its members. ${ }^{11}$, So far, so uncontroversial: the cutlery set represents no addition of being over and above the knife, the fork, and the spoon. Does extensionality follow? It does not. Suppose that a plurality just is some things as picked out by an expression with a particular sense $;{ }^{12}$ then extensionality will fail. This

\footnotetext{
${ }^{10}$ In private correspondence Linnebo has raised the possibility of extending groups to non-social cases of non-rigid collections. At this point the difference between his position and that supported in this paper becomes a subtle one. Many of what I think are pluralities, he thinks are groups. I do not think that an ontological commitment is incurred to anything over and above the members of the group, and I think that the surface grammar of the plural expressions typically used to denote these collections faithfully reflects their semantically plural status.

${ }^{11}$ It is a nice, and generally ignored question, how precisely the 'is' in this sentence should be understood. It looks like it cannot be an identity predicate on pain of violating type restrictions (and thereby introducting the threat of a form of Cantor's paradox).

${ }^{12}$ Objection: If a plurality is some things as picked out via a particular sense then it is not just some things. Reply on behalf of the position: The objection fails to take account of how central questions of ontological commitment are to the standard debate about plurals. I am claiming plural expressions do not carry ontological commitments not already implicit in singular
} 
would, amongst other things, open the door to the denial of the plurality/ group distinction, ${ }^{13}$ a move which in addition to ontological economy ${ }^{14}$ has in its favour the undoubted fact that many groups are picked out by noun-phrases linguistically indiscernible from plural NPs. At this point questions would likely be raised about whether commitment to pluralities is any less costly than commitment to objects [8].

Let's grant extensionality for pluralities, at least for the sake of argument: problems remain with the argument. It is taken for granted that extensionality is captured by (Indisc), where modal operators may occur within the substitutends for the sentential metavariables. Should somebody not antecedently disposed to accept $p n$ accept this characterisation of plural extensionality? Here is one reason why they ought not to: a non-singularist meaning theory for plural vocabulary could hold, consistently with the rejection of a parallel view for singular terms, that our referential practices with plurals are fine-grained, allowing the pluralities we track with them to come apart modally. After all, they might insist, openess to this kind of proposal is part of what it is to take plurals seriously. And this being so, allowing modal operators to be substituted within (Indisc) is question-begging.

None of this is decisive either way. Sceptical doubt has been introduced to Linnebo's plausibility argument, which ought perhaps to cause us to question whether we can formulate a logicometaphysical account of pluralities independent of developing a better understanding of the function of plural expressions in language. We'll return to that point in due course. Perhaps, though, we can short-circuit that imperative for present purposes. Might it not be that formal arguments for $p n$ are available, showing that the contested principle follows from commonly accepted (or at least plausible) rules governing plurals? Linnebo thinks so, and supplies three arguments to that effect. To these our attention now turns.

\section{2}

Here and in what follows, we will work in the logic PFO+ enriched with a singular existence predicate. For details of PFO+, see the appendix. We will allow ourselves the free use of modal operators, and leave the syntax of these intuitive. Linnebo's first argument for $p n$ we will call Uniform

terms referring to the constituent members of pluralities. This is prima facie compatible with attributing additional semantic content to plural expressions. It is up to my opponent to show otherwise.

${ }^{13}$ A group being a collection of people picked out by some role or feature, such as the Supreme Court justices. See [22].

${ }^{14}$ Although an alternative view, congruent with recent advocacy of easy ontology would concede the existence of groups, since identity conditions for groups can be supplied in terms of the corresponding pluralities, but deny their explanatory role [21]. 
Adjudication (UA). Versions of UA are supplied for PFO+ with and without an existence predicate. Since nothing important turns on which argument is at issue, we will focus on that without the existence predicate for the sake of simplicity.

Add to the language of PFO+ a dyadic plural term forming operator ' + ', which takes a singular term in its first argument place and a plural term in its second. The intended interpretation of this operator is as denoting adjunction, the operation of 'adjoining one object to a plurality' [13, 11]. The following seems plausible as a principle governing adjunction:

(UniAdj)

$$
\square \forall x(x<x x+a \leftrightarrow x<x x \vee x=a)
$$

Linnebo doesn't provide an argument for (Uniadj), but one may be easily be found. The principle is, after all plausibly understood as (implicitly) definitional of the adjunction operator, and as such analytic and therefore necessary. ${ }^{15}$ Given (UniAdj), the next stage in (UA) is to assume $a<x x$. This gives us $x x \equiv x x+a$ by (UniAdj). We are licensed to necessitate this by (Cov), yielding:

$$
\square(x x \equiv x x+a)
$$

The next stage is to argue that (UniAdj) yields:

$$
\square(a<x x+a)
$$

According to Linnebo (UniAdj) entails (2) within the scope of our assumption, but no proof is provided. Let's talk through how one might go. Instantiating the bound variable with $a$, application of $\mathbf{K}$ to the R-L direction of (UniAdj) delivers:

$$
\square(a \prec x x \vee a=a) \rightarrow \square(x x+a)
$$

The necessity of identity and modus ponens give us (2). Returning to (UA), with (2) under our belts, we can appeal to (1) and get $\square(a<x x)$. We discharge the assumption and we are done.

The problem with (UA) as a case for (NecInc) - remember that nothing here turns on the presence of an existence predicate - is the invocation of (Cov) to necessitate (1). Recall that (Cov) is derived using (Indisc):

\footnotetext{
${ }^{15}$ Those beset by Quinean scruples about analyticity are invited to reformulate this argument.
} 
(Indisc)

$$
x x \equiv y y \rightarrow(\phi(x x) \leftrightarrow \phi(y y))
$$

In the context of an argument for a component of $p n$ this looks problematic. In order to get the derivation of (Cov) we need modal vocabulary to be admissible in the substitutends for the sentential metavariables. But it is doubtful than anyone who doubts $p n$, and so anyone with worries about (NecInc) should allow this. For this admission expresses formally the doctrine that pluralities are extensional in a strong sense. Above we observed that the admission of modal vocabulary in instances of (Cov) begs questions about the constraints on the semantic behaviour of plurals in modal contexts. This is a defeating worry for the use of (Cov) in (UA). Someone not antecedently committed to pn ought only to admit substitutends in (Indisc) that do not contain modal operators. This is not enough to derive (Cov), so (UA) is not available to the non-pn believer. But then, in particular, it is not available when arguing with a non-pn believer; which is to say it lacks the required suasive force. ${ }^{16}$

The second argument is owing to Williamson, and termed by Linnebo partial rigidification (PR) [24, 699-700]. Considering a fixed domain context first, this proceeds from an instance of plural comprehension:

$$
\exists y y(x x \equiv y y \wedge \forall x(x<y y \rightarrow \square x<y y))
$$

$y y$ here is a partial rigidification of $x x$. Now assume $a \prec x x$ and consider $y y$, the partial rigidification of $x x$. This gives us $\square a<y y$. Together with $\square(x x \equiv y y)$, which we have by (Cov), the derivation of $\square a \prec x x$ is immediate. Discharging the assumption gives us (NecInc).

Linnebo considers this persuasive, but of limited value, since the fixed domain assumption is unlikely to appeal to anyone not signed up to Williamsonian necessitism. ${ }^{17}$ I doubt even that (PR) is of use in arguing for pn even modulo a fixed domain, because like (UA) it appeals to (Cov), and as

\footnotetext{
${ }^{16}$ Similar considerations tell against the admission of modal vocabulary in the comprehension schema appealed to in $[23$, 237]

${ }^{17}$ On which, see [25].
} 
we have seen this looks question-begging. Things look unfavourable also when (PR) is adapted for variable domains. To do this, we use a different instance of plural comprehension:

$$
\exists y y(x x \equiv y y \wedge \forall x[x<y y \rightarrow \square(E y y \rightarrow \square x \prec y y)])
$$

We proceed in a similar fashion as for the fixed-domain case, this time concluding that $\square(E y y \rightarrow$ $a \prec y y$ ) on the assumption that $a \prec x x$. Appealing to (Cov) would get us $\square(E x x<a<x x$ ), allowing us to discharge and get (NecInc) only given that $\square(E x x \rightarrow E y y)$, but as Linnebo himself acknowledges this is not something that somebody who rejects $p n$ is likely to allow. If one is prepared to grant that $y y$ are actually all and only $x x$, but contingently so, what stands in the way of granting the possibility that $y y$ exist but $x x$ do not?

For this reason Linnebo takes (PR) to have force against the denier of $p n$ in a fixed domain, but not in a variable domain context. He is certainly correct regarding the latter case, but it is unclear that the argument is any more convincing when the domain is fixed. As we saw, in that case (PR) still relies on $(\mathrm{Cov})$, and at this point the pn-denier ought to refuse to follow. We arrive here at a theme recurring throughout the present paper; formal arguments for $p n$ are less successful than even Linnebo, a sober and balanced commentator, allows. The principle does not appear susceptible to proof from premises which are not too close to the principle itself for the proof to have suasive force. This will become more apparent when we consider the third and final of Linnebo's formal arguments.

Say that a plurality is traversable just in case its members can be exhaustively listed. This is straightforward in the case of a finite plurality. For example, a three-membered plurality aa might be traversed as follows:

$$
\forall x(x<a a \leftrightarrow x=a \vee x=b \vee x=c)
$$


Linnebo says that we can assert uniform traversability, by which is meant the necessitation of wffs such as $((6)),{ }^{18}$ thus,

$$
\square \forall x(x<x x \leftrightarrow x=a \vee x=b \vee x=c)
$$

In the context of an argument for $p n$ this might look fatally question-begging, a point to which we'll return in due course. First we need to deal with the case of infinite pluralities. Here substantial infinitary resources are required. ${ }^{19}$ Working in plural $\mathcal{L}_{\infty \omega}$, and adopting the notation of [10], uniform traversability in this case may be stated with $i$ ranging over sufficient ordinals to index the members of $x x$

(NDIS)

$$
\square\left(x<x x \leftrightarrow \bigvee_{\forall i}\left(x=a_{i}\right)\right)
$$

Given $\mathbf{T}$ in the background modal logic we can prove ${ }^{20} \square y<x x$ on the assumption that $y<x x$, giving us (NecInc) for fixed domains. A similar argument is forthcoming in when an existence predicate is predicate, giving us (NecInc) simpliciter. However, as Linnebo himself admits,

[the] premise of universal traversability is little other than an infinitary restatement of our target claim that a plurality is fixed in its membership as we shift our attention from one possible world to another. $[13,14]$

As advertised, there is little reason to believe that an assertion of uniform traversability will do anything to convince anyone not antecedently given to assent to $p n$. This much Linnebo himself concedes. However, the other formal arguments he deploys are in equally bad shape as we have seen. In each case the complaint is the same: the argument will not persuade somebody not already disposed to assent to $p n$ since at a crucial stage it deploys an argument that such a person has no reason to accept. We know that (NecInc), and therefore $p n$, is independent of any normal modalisation of PFO+, so to the extent that these logics capture pre-formal reasoning about plurals and modality our target doesn't fall out of principles governing thought. What is more, $p n$ has been questioned by a minority of philosophers. Still, my impression is that the great majority assent to it. Given the use to which the principle is put in philosophy, it would be unfortunate if it lacked adequate justification; yet the position we have reached suggests that this might be a live possibility. Can more be said?

\footnotetext{
${ }^{18}$ Note that the principle of uniform traversability for finite pluralities is not statable in the object language.

${ }^{19}$ The language of $\mathcal{L}_{\infty \omega}$ expands that of first-order logic with identity by admitting disjunctions and conjunctions of any transfinite length, whilst allowing only finite blocks of quantifiers. We assume, moreover, that the language is equipped with a proper class of individual constants.

${ }^{20}$ For details see $[10$,$] .$
} 
We might be tempted to think that too much is being conceded to scepticism. After all, a plural variable ' $x x$ ' is supposed to formalise a natural language expression such as 'these things'. But, one can imagine an exasperated interlocutor protesting, if this thing is one of these things then of course it is necessarily the case that this is one of these things, or else they wouldn't be these things. Unfortunately, this is simply an affirmation of faith in place of an argument: the claim that 'these things' designates all and only these things with respect to a counterfactual situation is implicit in 'or else they wouldn't be these things'. The expression 'these things' is being used to justify the claim that of necessity these things are all and only these things. Yet it can only do this on assumption that 'these things' is semantically rigid in the sense that ${ }^{21}$ if it designates anything with respect to a counterfactual situation then it designates all and only the things it actually designates. This semantic presupposition is not $p n$, to identify the two would be a gratuitous confusion of use and mention; however it is clearly closely related. As we shall now see.

The formal logic of plurals is of interest because it allows us to codify plural concepts we express in natural language and to capture the rules of deduction governing these. Were PFO+ merely a mathematical system, understood formalistically, then there would be no barrier to importing any interaction with modal operators we care to mention in order to extend the system. For as long as our concern is mere symbolic games, one game is as good as another. But on the intended interpretation of PFO+ as a plural logic, the metatheoretic statement which appropriately formalises the claim that $\alpha \alpha$ is semantically rigid just in case if it designates anything with respect to a counterfactual situation then it designates all and only the things it actually designates is supposed to model a claim that could be made about natural language plurals. And a meaning theory for a natural language should be statable in (the same) natural language itself, worries about the semantic paradoxes notwithstanding. ${ }^{22}$ But now it is clear that the move to the metalanguage imposes no rigidity on object language plurals that wasn't already implicit in the statement of object language $p n$ itself, for

\footnotetext{
${ }^{21}$ The term '(semantically) rigid' used of a plural NP is dangerously ambiguous. My usage here has it that $\alpha \alpha$ is rigid iff (a) if $x$ is one of the things designated by $\alpha \alpha$ then $x$ is one of the things designated by $\alpha \alpha$ with respect to any world at which $\alpha \alpha$ designates some things and $x$ exists; and (b) if $x$ is not one of the things designated by $\alpha \alpha$ then $x$ is not one of the things designated by $\alpha \alpha$ with respect to any world at which $\alpha \alpha$ designates some things and $x$ exists. An alternative usage would have it that simply $\alpha \alpha$ is rigid iff $\alpha \alpha$ designates the same plurality with respect to any world at which $\alpha \alpha$ designates any plurality. My view is that latter claim is utterly harmless, indeed uninformative, since we have no grasp of what pluralities are, what it is for some things to be considered as many, apart from our use of plural language. Pluralities just are what plural expressions designate.

${ }^{22}$ I agree with Priest about this much, although dissent from his dialethic response to paradox [16]. Rather I would want to follow Rumfitt in maintaining that paradoxical sentences do not succeed in making statements [20,].
} 
ultimately the semantic principle for the formal language should impose nothing not present in the natural language which is our object of investigation. In that language: 'these things', used in the statement of a theory of meaning for the language, means these things. The constraint on a meaning theory that it licence the disquotational statement of the semantic value of lexical items entails that the appeal to natural language in the fashion proposed by the idignant objector of the previous paragraph gives no new suasive purchase.

Where does this leave us with respect to $p n$ ? If a widely accepted logic of plurals doesn't deliver $p n$ as a theorem, no matter which (normal) modal logic it is combined with, and if obvious formal arguments departing from the accentuation of plural logic with metaphysico-semantic principles concerning plurals, what possible motivation could there be for adopting $p n$ ? A temptation at this point is to appeal to more robustly metaphysical considerations about the nature of pluralities. Provisional on metaphilosophical choices minuted below, this gives the impression of an admission of defeat under the guise of further enquiry. For whatever the nature of pluralities might be, it is surely something that we approach through our canonical means of latching on to them, that is through the use of plural language. How else can I explore what it is to be a plurality other than through attention to our plural talk and reasoning? Do not be misled by comparisons with the scientific study of entities of some particular class. I can explore the nature of water through using an electron microscope: my use, notoriously, will not settle the question. But 'plurality' is not a sortal of the same kind as 'water': it expresses a general, formal, concept applicable across a wide range of collections of entities of all sorts and categories. In its formality it belongs with 'object', rather than 'positron'; and the prospects for a substantial enquiry into the nature of objects, as opposed to a logico-semantic making lucid of the place of objects in the structure of the world as approached through language, are not good.

We cannot step outside of language to study the general structure of the world in its metaphysical purity, whatever that would involve. Such is our logocentric predicament. ${ }^{23}$ What is it about the use of plural terms - 'these students', 'the Channel Islands', 'Pixies' (if we take the last to be semantically plural, and not a singular name for a group - that licences the application of $p n$ ? This, it seems to me, is the only question that could give us a good reason to adopt the principle. And here

\footnotetext{
${ }^{23}$ For the record, I don't see it as a much of a predicament. It seems to be a serious problem for as long as we are caught up in a picture according to which language somehow distorts our access to reality. But the picture is not compulsory. We can, alternatively, view language as our means to grasp reality conceptually, to make its contents the objects of reasoning. As Dummett puts it, 'language may be a distorting mirror, but it is the only mirror we have' $[6,6]$.
} 
there nothing in use that proponents of the principle have thus far rallied to its support, except with respect to the proper sublplurality of plural terms consisting of compound names - 'Ant and Dec', ' 2 and 17 ' - and these are not the contested cases. ${ }^{24}$ Moreover, there is some evidence from usage against $p n$ : 'You should be careful what you are saying; Smith could have been one of those men', to adapt an example of Dorothy Edgington's. ${ }^{25}$ To complain that here 'those men' is associated with descriptive content ('the men talking in that corner at this party') is simply to shift the dialectic, and not in a manner that looks likely to favour $p n$. Are plural terms associated with some kind of intensional content, a sense distinct from their reference? What would decide this issue? A prime datum here seems to be precisely the kind of case Edgington proposes,${ }^{26}$ and this supports not $p n$ but its negation, since it provides a prima facie example of an instance for which the universal claim fails.

I do not take this to be decisive. Perhaps there is an argument to be had in favour of $p n$ that answers this objection. It might be, moreover, that reasonable constraints on a meaning theory motivate acceptance of the contested principle: what happens, for example, if we formalise modal plural logic along natural deduction lines, imposing the familiar requirements of harmony and conservativity on rules for logical vocabulary and treating plural terms correspondingly in an inferentialist fashion ${ }^{27}$ The point is merely that the work of motivating $p n$ philosophically remains to be done, and that in the absence of such work the appeal to the principle in support of strong metaphysical theses is illegitimate.

The frustrated defender of $p n$ has another avenue of response. Isn't to seek to ascertain the truth of the matter as regards pn, as we have been doing, in isolation from the project of developing our best overall theory of the world to succumb to methodological error? If the benefits of adding pn to one's theoretical arsenal outweigh the costs, for example in terms of sitting uncomfortably with current usage, then one should adopt the principle. This after, all is how science proceeds, and metaphysics

\footnotetext{
${ }^{24}$ In many cases, the reference of 'we' is anaphoric for that of a compound name. Unfortunately this makes the cute example with which the present paper began something of a ladder to be taken away once the dialectic is going.

${ }^{25}$ Cited in $[19,120-1]$.

${ }^{26}$ Note that we are dealing here with a plural demonstrative, so comparisons with singular descriptions such as 'the number of planets' to motivate the thought that 'those men' is not a genuine term are illicit. Against the objection that the sortal supplies descriptive content we can reply that (a) it doesn't supply enough content to secure pn, and (b) There is a case to be made that all terms, including singular names, are associated with sortals $[21],[14$,$] , so this line of objection is in danger of$ generalising uncontrollably.

${ }^{27}$ This would be moderately surprisingly, since the inferential rules would have to produce a distinct logic from PFO+ or any of its subsystems, of which (NecInc) is provably independent. To the best of my knowledge, however, no work has been done on natural deduction formulations of plural logic, although it should prove to be a routine exercise involving the adaptation of a formulation of second-order logic. My point in mentioning the inferentialist appeal to rules is not to convey an optimism that progress might be obtained by this route, but rather to indicate the kind of project a result from which would provide an acceptable resolution of the present debate.
} 
and logic are part of total science. Thus, for example, Williamson has stressed the methodological continuity between 'modal logic as metaphysics' and science $[25,422-429] .{ }^{28}$ This position is, to my mind, unconvincing. The disanalogies between metaphysics and the natural sciences are legion, most obviously in the failure of metaphysical theories to make empirically testable predictions. It might be retorted that metaphysical theories can be tested against intuitions, perhaps by means of thought experiments, these providing something analogous to the experiments of natural scientists. The role of intuitions in philosophy has been subject to a good deal of critical scrutiny of late ([7], [5]) and the analogy between thought experiments and experiments simpliciter has been questioned, rightly in my view ([9]). However, even within the general methodological framework in question there would seem to be particular concerns about appeals to intuition to support pn. For here, if we are not to confine our attention to imagined scenarios of the Edgington type (which hardly offer unambiguous support to $p n$ ) we will be arguing about intuitions and /or counterfactual judgements concerning either the truth of sentences expressed using English plural terminology ('In this situation, this would be one of these') or philosophical terms of art ('plurality'). In the first case, the above mooted accusation of circularity recurs; in the latter, the supposed role of intuition recedes into the background, and we are left simply with a priori metaphysical disputation of the old school, with no obvious similarity to the natural sciences or any other uncontroversially truth-conducive enterprise. Either way, moreover, there is a real danger of dominant views amongst current metaphysicians on $p n$ reinforcing themselves by appeal to the intuitions of the already-convinced, a procedure that is of no epistemic value.

If what I have suggested above is correct with respect to $p n$ then it looks likely to generalise beyond the case of $p n$ to other modal intuitions to which common and important appeal is made in philosophy. It is commonplace for $\mathbf{S 5}$, or at least $\mathbf{S} \mathbf{4}$ to be appealed to as the 'right' modal logic, and for metaphysical investigation to proceed with one of these as the background modal logic. There are disimilarities with the case of $p n$ : notably appeals to model theoretic semantics and to counterfactuals in support of S5. In both cases, I contend that circularity worries similar to those noted about arguments for $p n$ arise. Establishing that will have to wait for a subsequent paper. What is clear, however, is that in the absence of these arguments support for strong modal logics will rest on

\footnotetext{
${ }^{28}$ More recently he has appealed directly to data from natural science in support of modal claims: specifically dynamic systems theory is rallied to make a case for the Barcan principle and its converse [26]. There is a lot to be said about this, but in any case it is not clear how the move could be generalised to support plural necessitism.
} 
judgements concerning iterated modalities that are at least as precarious as those concerning expressions of $p n$ in natural language. Perhaps we should just be more modest in our claims about modality.

\section{APPENDIX A. LANGUAGE}

The logic PFO+ extends first-order logic with identity. To the lexicon we add:

- Denumerably many plural variables: ' $x x_{1}$ ', ' $x x_{2}$ '...

- Denumerably many plural constants: ' $a a_{1}$ ', ' $a a_{2}$ '...

- Denumerably many (monadic) plural predicates: ' $F F_{1}$ ', ' $F F_{2}$ '...

- The logical dyadic predicate ' $<$ ', which we read 'is one of'.

Customary abbreviations and modifications of notation are allowed. We add new formation rules:

A.1. Atomic wffs. Where $t$ ranges over singular, and $t t$ plural, terms:

- $\ulcorner t<t t\urcorner$ is a wff.

A.2. Molecular wffs. Where $\phi$ is a wff, $v v$ a plural variable, and with the usual constraints to avoid clash of variables:

- $\ulcorner\exists v v \phi\urcorner$ is a wff.

- $\ulcorner\forall v v \phi\urcorner$ is a wff.

Nothing other than elements of the closure of the atomic wffs under the formation rules is a wff.

\section{APPENDiX B. PROOF SYSTEM}

To any proof system for first-order logic with identity we add the axiom:

Nonemptiness: $\forall x x \exists y y<x x$

And the axiom schema:

Comprehension: $\exists x \phi \rightarrow \exists x x \forall x(x<x x \leftrightarrow \phi)$

We can also introduce the defined dyadic plural predicate ' $\equiv$ ', which we read 'are the same things as': 
Extensionality: $\forall x x \forall y y x x \equiv y y \leftrightarrow \forall x(x<x x \leftrightarrow x<y y)$

\section{APPENDIX C. MODEL-THEORETIC SEMANTICS}

Following [10] we will develop a model theoretic semantics for the language of PFO+. We will restrict our attention to fixed domains and show that, even with this restriction, (NecInc) is not valid. We will then add an existence predicate and consider variable domains. We will see that this does not affect the result.

Let a frame $\mathcal{F}=(S, R)$, with $S$ non-empty and $R$ a relation on $S$. A model $\mathcal{M}$ on $\mathcal{F}$ is $\mathcal{M}=$ $(S, R, D, I)$, with $D$ non-empty and $I$ a function that makes assigments to the non-logical vocabulary:

- To each singular constant some $d \in D$.

- To each $n$-adic predicate, for each $s \in S$, some $d \subseteq D^{n}$.

- To each plural constant, a non-empty set $(x, y) x, y \in P$ s.t. $\forall(x, y) \in P x \in S \wedge y \subseteq D \wedge \forall z(<$ $x, z) \in P \rightarrow z=y)$

- To each plural predicate, for each $s \in S$ some $p \subseteq \wp(D)$.

Given a model, we define a valuation function $v$, which assigns to each singular variable $x$, $v(x) \in D$ and to each plural variable $x x, v(x x) \subseteq S \times(D-\emptyset)$, s.t. $\forall s \in S((s, a) \in v(x x) \wedge(s, b) \in$ $v(x x)) \rightarrow a=b$.

Satisfaction can be defined. Writing $\mathcal{M}, s \Vdash_{v} \phi$ to indicate that $v$ and $\mathcal{M}$ satisfy $\phi$ at $s$ we proceed as follows, with $t$ ranging over singular terms.

(1) For an n-adic predicate ' $F$ ', $\mathcal{M}, s \Vdash_{v} F t_{1} \ldots t_{n}$ iff $\left(v\left(t_{1} \ldots v\left(t_{n}\right)\right) \in I(F, s)\right.$.

(2) For a plural constant ' $a a$ ', $\mathcal{M}, s \Vdash_{v} t<a a$ iff.$\exists(m, n) \in I(a a)(m=s \wedge t \in n)$

(3) For a plural variable ' $x x$ ', $\mathcal{M}, s \Vdash_{v} t \prec x x$ iff $\exists(m, n) \in v(x x)(m=s \wedge t \in n)$.

(4) For a plural predicate ' $F F$ ' and a plural constant ' $a a$ ', $\mathcal{M}, s \Vdash_{v} F F a a$ iff $\exists(m, n) \in I(a a)(m=$ $s \wedge n \in I(F F, s)$.

(5) For a plural predicate ' $F F$ ' and a plural variable ' $x x$ ', $\mathcal{M}, s \Vdash_{v} F F$ aa iff $\exists(m, n) \in v(x x)(m=$ $s \wedge n \in I(F F, s)$. 
Compound wffs are dealt with recursively:

(1) $\mathcal{M}, s \Vdash_{v} \neg \phi$ iff $\mathcal{M}, s \nVdash_{v} \phi$.

(2) $\mathcal{M}, s \Vdash_{v}(\phi \wedge \psi)$ iff $\mathcal{M}, s \Vdash_{v} \phi$ and $\mathcal{M}, s \Vdash_{v} \psi$.

(3) $\mathcal{M}, s \Vdash_{v} \square \phi$ iff $\forall u \in S$ if $s R u$ then $\mathcal{M}, u \Vdash_{v} \phi$.

(4) $\mathcal{M}, s \Vdash_{v} \forall x \phi$ iff for every valuation $w$, which differs from $v$ at most with respect to the assignment to ' $x$ ', $\mathcal{M}, s \Vdash_{w} \phi$.

(5) $\mathcal{M}, s \Vdash_{v} \forall x x \phi$ iff for every valuation $w$, which differs from $v$ at most with respect to the assignment to ' $x x^{\prime}, \mathcal{M}, s \Vdash_{w} \phi$

A wff is true in $\mathcal{M}$ if it is satisfied on every assignment at every world in $\mathcal{M}$. A wff is valid relative to a class of frames, if it is true in every model based on a frame from the class. A wff is valid simpliciter if it is true in every model. The concepts of truth and validity can be extended from wffs to theories in the natural way. Consequence is similarly defined naturally.

Theorem 1. (NecInc) is not $\boldsymbol{K}$-valid.

Proof. Let $\mathcal{F}=(\{0,1\}, R)$. Now consider a model $\mathcal{M}$. Let $R=\{(0,1),(1,0)\}$ and let $D=\{\pi, e\}$. Consider a valuation $v$ including $v(x)=\pi$ and $v(x x)=\{(0,\{\pi\}),(1,\{e\})\}$. At 0, ' $x<x x$ ' is satisfied, but ' $\square x \prec x x$ ' is not, since $0 R 1$ and $\mathcal{M}, 1 \nVdash_{v} x \prec x x$. Hence (NecInc) is not satisfied at 0 . It follows that (NecInc) is is invalid in $\mathcal{M}$, and thus that it is $\mathbf{K}$-invalid.

The soundness of the semantics for PFO+ is proved by a routine induction on the length of proofs and we omit the details.

\section{APPENDIX D. ADDING AN EXISTENCE PREDICATE}

To work with PFO+ in a variable domain context, we first of all add a special predicate ' $E$ ' to the lexicon s.t. $\ulcorner E t t\urcorner$ and $\ulcorner E t\urcorner$ are wffs ${ }^{29}$ We modify the axioms as follows:

V-Nonemptiness: $\forall x x(E x x \rightarrow \exists y(E y \wedge y<x x))$

Comprehension: $\exists x(E x \wedge \phi) \rightarrow \exists x x(E x x \wedge \forall x(x<x x \leftrightarrow(E x \wedge \phi)))$

We modify the model semantics so that $\mathcal{M}=(S, R, D, I, N) . N$ assigns to each world $s N_{s} \subseteq D$, the inner domain of $s$. We interpret ' $E$ ' s.t. ' $E t$ ' is satisfied at a world under a valuation iff $v(t) \in N_{s}$;

\footnotetext{
${ }^{29}$ We can think of ' $E$ ' in terms of two predicates, orthographically indiscernible, one singular and one plural. Alternatively,
} see footnote $v$ above. 
similarly for plural terms iff $v(t t) \subseteq N_{s}$. We can see that (NecInc) still fails to be valid: simply modify the above countermodel such that the inner domain is coextensive with the domain at each world.

$*$

\section{REFERENCES}

[1] Patrick Blackburn, Maarten de Rijke, and Yde Venema, Modal Logic, Cambridge University Press, Cambridge, 2002 Cambridge Tracts in Theoretical Computer Science 53.

[2] George Boolos, The iterative conception of set, in Logic, Logic and Logic [3], pp. 13-29.

[3] _ _ Logic, Logic and Logic, Harvard University Press, Cambridge, MA., 1998.

[4] , To be is to be a value of a variable (or to be some values of some variables), in Logic, Logic and Logic [3], pp. 54-72.

[5] Herman Cappelen (ed.), Philosophy Without Intuitions, Oxford University Press, Oxford, 2012.

[6] Michael Dummett, Origins of Analytical Philosophy, Bloomsbury, London, 2014.

[7] Matti Eklund, Intuitions, Conceptual Engineering, and Conceptual Fixed Points, Palgrave Handbook of Philosophical Methods (Chris Daly, ed.), 2017.

[8] Salvatore Florio and Øystein Linnebo, On the Innocence and Determinacy of Plural Quantification, Nous (2015), Forthcoming.

[9] Peter Hacker, A Philosopher of Philosophy: a review of Timothy Williamson's The Philosophy of Philosophy, Philosophical Quarterly 59 (2009), no. 235, 337-348.

[10] Simon Hewitt, Modalising plurals, Journal of Philosophical Logic 41 (2012), 853-875.

[11] Øystein Linnebo, Plural quantification, Article in the Stanford Encyclopedia of Philosophy, 2012, Available online at http://plato.stanford.edu/entries/plural-quant/ .

[12] _ The Potential Hierarchy of Sets, Review of Symbolic Logic 6 (2013), no. 2, 205-29.

[13] _ Plurals and Modals, Canadian Journal of Philosophy (2016), DOI: 10.180/00455091.2015.1132975.

[14] E.J. Lowe, Forms of Thought : a study in philoosphical logic, Cambridge University Press, Cambridge, 2013.

[15] Ruth Barcan Marcus, Modalities and intensional languages, Synthese 13 (1961), no. 4, 303-322.

[16] Graham Priest, In Contradiction, 1st ed., Martinus Nojhoff, Dordrecht, 1987.

[17] Graham Priest, Is the Ternary R Depraved?, Foundations of Logical Consequence (Colin R. Caret and Ole T. Hjortland, eds.), Oxford University Press, Oxford, 2015, pp. 121-135.

[18] Agustin Rayo, Plurals, Philosophical Compass 2 (2007), no. 3, 411-27.

[19] Ian Rumfitt, Plural terms: Another variety of reference?, Thought, Reference, and Experience : Themes from the Philosophy of Gareth Evans (J.L. Bermudez, ed.), Clarendon Press, Oxford, 2005, pp. 84-123.

[20] The Boundary Stones of Thought : an essay on the philosophy of logic, Oxford University Press, Oxford, 2015.

[21] Amie L. Thomasson, Ontology Made Easy, Oxford University Press, Oxford, 2015.

[22] Gabriel Uzquiano, The Supreme Court and the Supreme Court Justices : A Metaphysical Puzzle, Nous 38 (2004), no. 1, 135-153.

[23] _ Plural Quantification and Modality, Proceedings of the Aristotelian Society 111 (2011), 219-250. 
[24] Timothy Williamson, Necessitism, contingentism and plural quantification, Mind 119 (2010), no. 475, 657-748.

[25] , Modal Logic as Metaphysics, Oxford University Press, Oxford, 2013.

[26] _ Modal science, Canadian Journal of Philosophy 46 (2016), no. 4-5, 453-492.

* School of Religion, History and the Philosophy of Science, University of LeEds, LS2 9JT. UK.

E-mail address, S. Hewitt: s.hewitteleeds.ac.uk 